\title{
Leukemia mathematical model
}

\author{
Nacera Bouizem ${ }^{1, \mathrm{a}}$, Mohamed Helal ${ }^{1, \mathrm{~b}}$, Bedr'Eddine Ainseba ${ }^{2, \mathrm{c}}$, and Abdelkader Lakmeche ${ }^{1, \mathrm{~d}}$ \\ ${ }^{1}$ Laboratory of Biomathematics, Univ. Sidi Bel Abbes, PB. 89, Sidi Bel Abbes 22000, Algeria \\ 2 IMB, Université Bordeaux Victoire, 3 ter place de la Victoire-Case 36, 33076 Bordeaux Cedex, \\ France
}

\begin{abstract}
In this paper, we study a mathematical model of leukemia diseases. We find sufficient conditions for existence and local stability of steady states.
\end{abstract}

\section{Introduction}

Hematopoietic stem cells are found in the bone marrow and are able to renew themselves through cell division producing blood cells, a significant increase in the number of white blood cells disease causes chronic myeloid leukemia which is characterized by a chromosomal anomaly acquired, that is the translocation between chromosome 9 and 22 giving birth to an abnormal chromosome called the Philadelphia chromosome, this translocation generates a protein from the merger of the Bcr gene on chromosome 22 and Abl genes on chromosome 9, this protein is a tyrosine kinase Bcr-Abl. Generally, tyrosine kinases are components that control cell proliferation, differentiation and apoptosis, they have a very important role in signal transduction because the tyrosine kinase can transfer a phosphate group from adenosine triphosphate to another protein in a cell but the tyrosine kinase Bcr-Abl instructs cells to grow out of control and prevents them from undergoing apoptosis, resulting in the formation of a tumor. Among the treatments can produce a significant chance of cure is IMATINIB [5] which is a competitive inhibitor of the tyrosine kinase activity because it will bind to quote binding of adenosine tri-phosphate prevents tyrosine to give these orders cancerous proliferation. The first mathematical models describing the dynamics of hematopoietic stem cells have been proposed by Mackey [3]. In recent works Adimy \& Crauste [1], Dingli \& Michor [2] and Michor et al. [4] have studied the dynamics of normal and cancer stem cells in chronic myeloid leukemia.

In our work we focus on the development of normal, cancerous and resistant hematopoietic stem cells in chronic myeloid leukemia. We study the existence of equilibrium points, their local stability and we give some numerical simulations.

\footnotetext{
a e-mail: nbouizem@yahoo.com

b e-mail: mhelal_abbes@yahoo.fr

c e-mail: bedreddine. ainseba@math.u-bordeaux1.fr

d e-mail: lakmeche@yahoo.fr
}

This is an Open Access article distributed under the terms of the Creative Commons Attribution License 4.0, which permits unrestricted use, distribution, and reproduction in any medium, provided the original work is properly cited. 


\section{The model}

We consider the following system

with

$$
\left\{\begin{array}{l}
x_{0}^{\prime}=n \Phi\left(x_{0}+y_{0}+z_{0}\right) x_{0}-d_{0} x_{0} \\
x_{1}^{\prime}=r x_{0}-\left(d-d_{2}\right) x_{1} \\
y_{0}^{\prime}=m \Psi\left(x_{0}+\alpha y_{0}+\alpha z_{0}\right) y_{0}-g_{0} y_{0} \\
y_{1}^{\prime}=q y_{0}-\left(g-g_{2}+h_{2}(u)\right) y_{1} \\
z_{0}^{\prime}=m \Psi\left(x_{0}+\alpha y_{0}+\alpha z_{0}\right) z_{0}-g_{0 r} z_{0} \\
z_{1}^{\prime}=q z_{0}-\left(d_{r}-d_{2 r}+h_{2 r}(u)\right) z_{1}
\end{array}\right.
$$

$$
\begin{aligned}
& \Phi\left(x_{0}+y_{0}+z_{0}\right)=\frac{1}{1+c_{x}\left(x_{0}+y_{0}+z_{0}\right)}, \quad \Psi\left(x_{0}+\alpha y_{0}+\alpha z_{0}\right)=\frac{1}{1+c_{y}\left(x_{0}+\alpha y_{0}+\alpha z_{0}\right)} \\
& g_{0 r}<g_{0} \\
& h_{2}, h_{2 r}:\left[0, u_{\max }\right) \longrightarrow[0,1) \\
& h_{2} \nearrow, h_{2 r} \nearrow, h_{2}(0)=0=h_{2 r}(0)
\end{aligned}
$$

where

\begin{tabular}{|c|l|}
\hline The parameters & meaning \\
\hline$n$ & division rate of normal stem cells \\
\hline$d_{0}$ & death rate of normal stem cells \\
\hline$r$ & production rate of normal differentiated cells \\
\hline$d$ & death rate of normal Differentiated cells \\
\hline$d_{2}$ & proliferation rate of normal differentiated cells \\
\hline$m$ & division rate of cancerous stem cells \\
\hline$\alpha$ & competitif coefficient \\
\hline$g_{0}$ & death rate of sensitive cancerous stem cells \\
\hline$q$ & production rate of cancerous differentiated cells \\
\hline$g$ & death rate of sensitive cancerous differentiated cells \\
\hline$g_{2}$ & proliferation rate of sensitive cancerous differentiated cells \\
\hline$h_{2}(u)$ & IMATINIB effect on sensitive cancerous differentiated cells \\
\hline$g_{0 r}$ & death rates of resistant cancerous stem cells \\
\hline$d_{r}$ & death rate of resistant cancerous differentiated cells \\
\hline$d_{2 r}$ & proliferation rate of resistant cancerous differentiated cells \\
\hline$h_{2 r}(u)$ & IMATINIB effect on resistant cancerous differentiated cells \\
\hline$u$ & drug dose administered \\
\hline
\end{tabular}

Table of parameters

and

\begin{tabular}{|c|l|}
\hline The variables & meaning \\
\hline$x_{0}$ & number of normal hematopoietic stem cells \\
\hline$y_{0}$ & number of sensitive cancerous hematopoietic stem cells \\
\hline$z_{0}$ & number of resistant cancerous hematopoietic stem cells \\
\hline$x_{1}$ & number of normal differentiated cells \\
\hline$y_{1}$ & number of sensitive cancerous differentiated cells \\
\hline$z_{1}$ & number of resistant cancerous differentiated cells \\
\hline
\end{tabular}

Table of variables 


\section{Existence and stability of equilibrium}

In this section, we analyze the existence and local stability of feasible equilibrium of (1).

\subsection{Existence of equilibrium}

The equilibrium points of (1) are

$$
\begin{aligned}
& \mathbb{E}_{0}=(0,0,0,0,0,0), \\
& \mathbb{E}_{1}=\left(x_{0}^{1}, x_{1}^{1}, 0,0,0,0\right) \text { where } x_{0}^{1}=\frac{1}{c_{x}}\left(\frac{n}{d_{0}}-1\right) \text { and } x_{1}^{1}=\frac{r}{\left(d-d_{2}\right)}\left[\frac{1}{c_{x}}\left(\frac{n}{d_{0}}-1\right)\right], \\
& \mathbb{E}_{2}=\left(x_{0}^{2}, x_{1}^{2}, y_{0}^{2}, y_{1}^{2}, 0,0\right) \text { where } x_{0}^{2}=\frac{1}{(1-\alpha)}\left[\frac{1}{c_{y}}\left(\frac{m}{g_{0}}-1\right)-\frac{\alpha}{c_{x}}\left(\frac{n}{d_{0}}-1\right)\right], \\
& x_{1}^{2}=\frac{r}{\left(d-d_{2}\right)(1-\alpha)}\left[\frac{1}{c_{y}}\left(\frac{m}{g_{0}}-1\right)-\frac{\alpha}{c_{x}}\left(\frac{n}{d_{0}}-1\right)\right], y_{0}^{2}=\frac{1}{(1-\alpha)}\left[\frac{1}{c_{x}}\left(\frac{n}{d_{0}}-1\right)-\frac{1}{c_{y}}\left(\frac{m}{g_{0}}-1\right)\right] \text { and } \\
& y_{1}^{2}=\frac{q}{\left(g-g_{2}+h_{2}(u)\right)(1-\alpha)}\left[\frac{1}{c_{x}}\left(\frac{n}{d_{0}}-1\right)-\frac{1}{c_{y}}\left(\frac{m}{g_{0}}-1\right)\right], \\
& \mathbb{E}_{3}=\left(x_{0}^{3}, x_{1}^{3}, 0,0, y_{0}^{3}, y_{1}^{3}\right) \text { where } x_{0}^{3}=\frac{1}{(1-\alpha)}\left[\frac{1}{c_{y}}\left(\frac{m}{g_{0 r}}-1\right)-\frac{\alpha}{c_{x}}\left(\frac{n}{d_{0}}-1\right)\right], \\
& x_{1}^{3}=\frac{r}{\left(d-d_{2}\right)(1-\alpha)}\left[\frac{1}{c_{y}}\left(\frac{m}{g_{0 r}}-1\right)-\frac{\alpha}{c_{x}}\left(\frac{n}{d_{0}}-1\right)\right], z_{0}^{3}=\frac{1}{(1-\alpha)}\left[\frac{1}{c_{x}}\left(\frac{n}{d_{0}}-1\right)-\frac{1}{c_{y}}\left(\frac{m}{g_{0 r}}-1\right)\right] \text { and } \\
& z_{1}^{3}=\frac{q}{\left(d_{r}-d_{2 r}+h_{2 r}(u)\right)(1-\alpha)}\left[\frac{1}{c_{x}}\left(\frac{n}{d_{0}}-1\right)-\frac{1}{c_{y}}\left(\frac{m}{g_{0 r}}-1\right)\right], \\
& \mathbb{E}_{4}=\left(0,0, y_{0}^{4}, y_{1}^{4}, 0,0\right) \text { where } y_{0}^{4}=\frac{1}{\alpha c_{y}}\left(\frac{m}{g_{0}}-1\right) \text { and } y_{1}^{4}=\frac{q}{g-g_{2}+h_{2}(u)}\left[\frac{1}{\alpha c_{y}}\left(\frac{m}{g_{0}}-1\right)\right], \\
& \mathbb{E}_{5}=\left(0,0,0,0, z_{0}^{5}, z_{1}^{5}\right) \text { where } z_{0}^{5}=\frac{1}{\alpha c_{y}}\left(\frac{m}{g_{0 r}}-1\right) \text { and } z_{1}^{5}=\frac{q}{d_{r}-d_{2 r}+h_{2 r}(u)}\left[\frac{1}{\alpha c_{y}}\left(\frac{m}{g_{0 r}}-1\right)\right] .
\end{aligned}
$$

Theorem 3.1: The system (1) admits feasible equilibrium points according to the following conditions.

1. The trivial point $\mathbb{E}_{0}$ exists always.

2. If $d_{0}<n$ and $d_{2}<d$ the equilibrium point $\mathbb{E}_{1}$ exists.

3. If $d_{2}<d, g_{2}<g+h_{2}(u), g_{0}<m$ and $\frac{\alpha c_{y} g_{0} n}{\alpha c_{y} g_{0}+c_{x}\left(m-g_{0}\right)}<d_{0}<\frac{c_{y} g_{0} n}{c_{y} g_{0}+c_{x}\left(m-g_{0}\right)}$ the equilibrium point $\mathbb{E}_{2}$ exists.

4. If $d_{2}<d, d_{2 r}<d_{r}+h_{2 r}(u), g_{0 r}<m$ and $\frac{\alpha c_{y} g_{0 r} n}{\alpha c_{y} g_{0 r}+c_{x}\left(m-g_{0 r}\right)}<d_{0}<\frac{c_{y} g_{0 r} n}{c_{y} g_{0 r}+c_{x}\left(m-g_{0 r}\right)}$ the equilibrium point $\mathbb{E}_{3}$ exists.

5. If $g_{0}<m$ and $g_{2}<g+h_{2}(u)$ the equilibrium point $\mathbb{E}_{4}$ exists.

6. If $g_{0 r}<m$ and $d_{2 r}<d_{r}+h_{2 r}(u)$ the equilibrium point $\mathbb{E}_{5}$ exists.

\subsection{Local stability of equilibrium}

The general form of the Jacobian with respect to each equilibrium point is given by Local Stability of equilibrium points 


$$
\mathcal{J}_{\mathbb{E}_{i}}=\left(\begin{array}{cccccc}
n \Phi-d_{0}+n x_{0}^{i} \frac{d \Phi}{d x_{0}^{i}} & 0 & n x_{0}^{i} \frac{d \Phi}{d y_{0}^{i}} & 0 & n x_{0}^{i} \frac{d \Phi}{d z_{0}^{i}} & 0 \\
r & d_{2}-d & 0 & 0 & 0 & 0 \\
m y_{0}^{i} \frac{d \Psi}{d x_{0}^{i}} & 0 & m \Psi-g_{0}+m y_{0}^{i} \frac{d \Psi}{d y_{0}^{i}} & 0 & m y_{0}^{i} \frac{d \Psi}{d z_{0}^{i}} & 0 \\
0 & 0 & q & g_{2}-g-h_{2}(u) & 0 & 0 \\
m z_{0}^{i} \frac{d \Psi}{d x_{0}^{i}} & 0 & m z_{0}^{i} \frac{d \Psi}{d y_{0}^{i}} & 0 & m \Psi-g_{0 r}+m z_{0}^{i} \frac{d \Psi}{d z_{0}^{i}} & 0 \\
0 & 0 & 0 & 0 & q & d_{2 r}-d_{r}-h_{2 r}(u)
\end{array}\right) .
$$

We have

$$
\operatorname{det}\left(\mathcal{J}_{\mathbb{E}_{i}}-\lambda \mathrm{I}\right)=\left(d_{2 r}-d_{r}-h_{2 r}(u)-\lambda\right)\left(g_{2}-g-h_{2}(u)-\lambda\right)\left(d_{2}-d-\lambda\right) \operatorname{det} C_{i}
$$

where

$$
C_{i}=\left(\begin{array}{ccc}
n \Phi-d_{0}+n x_{0}^{i} \frac{d \Phi}{d x_{0}^{i}}-\lambda & n x_{0}^{i} \frac{d \Phi}{d y_{0}^{i}} & n x_{0}^{i} \frac{d \Phi}{d z_{0}^{i}} \\
m y_{0}^{i} \frac{d \Psi}{d x_{0}^{i}} & m \Psi-g_{0}+m y_{0}^{i} \frac{d \Psi}{d y_{0}^{i}}-\lambda & m y_{0}^{i} \frac{d \Psi}{d z_{0}^{i}} \\
m z_{0}^{i} \frac{d \Psi}{d x_{0}^{i}} & m z_{0}^{i} \frac{d \Psi}{d y_{0}^{i}} & m \Psi-g_{0 r}+m z_{0}^{i} \frac{d \Psi}{d z_{0}^{i}}-\lambda
\end{array}\right) .
$$

\section{Theorem 3.2:}

1. The equilibrium point $\mathbb{E}_{0}$ is asymptotically stable if $d_{2 r}<d_{r}+h_{2 r}(u), g_{2}<g+h_{2}(u)$, $d_{2}<d, n<d_{0}$ and $m<g_{0 r}$.

2. The equilibrium point $\mathbb{E}_{1}$ is asymptotically stable if $d_{2 r}<d_{r}+h_{2 r}(u), g_{2}<g+h_{2}(u)$, $d_{2}<d, d_{0}<n$ and $\frac{m c_{x} d_{0}}{c_{x} d_{0}+c_{y}\left(n-d_{0}\right)}<g_{0 r}$.

3. The equilibrium point $\mathbb{E}_{2}$ is unstable.

4. The equilibrium point $\mathbb{E}_{3}$ is unstable.

5. The equilibrium point $\mathbb{E}_{4}$ is unstable.

6. The equilibrium point $\mathbb{E}_{5}$ is asymptotically stable if $d_{2 r}<d_{r}+h_{2 r}(u), g_{2}<g+h_{2}(u)$, $d_{2}<d, g_{0 r}<m$ and $\frac{n \alpha c_{y} g_{0 r}}{\alpha c_{y} g_{0}+c_{x}\left(m-g_{0 r}\right)}<d_{0}$.

Proof:

1. For the equilibrium point $\mathbb{E}_{0}$ we have

$$
\mathcal{J}_{\mathbb{E}_{0}}=\left(\begin{array}{cccccc}
n-d_{0} & 0 & 0 & 0 & 0 & 0 \\
r & d_{2}-d & 0 & 0 & 0 & 0 \\
0 & 0 & m-g_{0} & 0 & 0 & 0 \\
0 & 0 & q & g_{2}-g-h_{2}(u) & 0 & 0 \\
0 & 0 & 0 & 0 & m-g_{0 r} & 0 \\
0 & 0 & 0 & 0 & q & d_{2 r}-d_{r}-h_{2 r}(u)
\end{array}\right)
$$

and

$$
\operatorname{det}\left(\mathcal{J}_{\mathbb{E}_{0}}-\lambda \mathrm{I}\right)=\left(d_{2 r}-d_{r}-h_{2 r}(u)-\lambda\right)\left(g_{2}-g-h_{2}(u)-\lambda\right)\left(d_{2}-d-\lambda\right) \operatorname{det} C_{0}
$$


where

$$
C_{0}=\left(\begin{array}{ccc}
n-d_{0}-\lambda & 0 & 0 \\
0 & m-g_{0}-\lambda & 0 \\
0 & 0 & m-g_{0 r}-\lambda
\end{array}\right) .
$$

We have

$$
\begin{aligned}
\operatorname{det}\left(\mathcal{J}_{\mathbb{E}_{0}}-\lambda \mathrm{I}\right)= & \left(d_{2 r}-d_{r}-h_{2 r}(u)-\lambda\right)\left(g_{2}-g-h_{2}(u)-\lambda\right)\left(d_{2}-d-\lambda\right) \\
& \left(n-d_{0}-\lambda\right)\left(m-g_{0}-\lambda\right)\left(m-g_{0 r}-\lambda\right) .
\end{aligned}
$$

Then, the eigenvalues are $\lambda_{1}=d_{2 r}-d_{r}-h_{2 r}(u), \quad \lambda_{2}=g_{2}-g-h_{2}(u), \quad \lambda_{3}=d_{2}-d$, $\lambda_{4}=n-d_{0}, \lambda_{5}=m-g_{0}$ and $\lambda_{6}=m-g_{0 r}$.

2. For the equilibrium point $\mathbb{E}_{1}$ we have

$$
\mathcal{J}_{\mathbb{E}_{1}}=\left(\begin{array}{cccccc}
d_{0}\left(\frac{d_{0}}{n}-1\right) & 0 & d_{0}\left(\frac{d_{0}}{n}-1\right) & 0 & d_{0}\left(\frac{d_{0}}{n}-1\right) & 0 \\
r & d_{2}-d & 0 & 0 & 0 \\
0 & 0 & \frac{m c_{x} d_{0}}{c_{x} d_{0}+c_{y}\left(n-d_{0}\right)}-g_{0} & 0 & 0 & 0 \\
0 & 0 & q & g_{2}-g-h_{2}(u) & 0 & 0 \\
0 & 0 & 0 & 0 & \frac{m c_{x} d_{0}}{c_{x} d_{0}+c_{y}\left(n-d_{0}\right)}-g_{0 r} & 0 \\
0 & 0 & 0 & 0 & q & d_{2 r}-d_{r}-h_{2 r}(u)
\end{array}\right)
$$

and

$$
\operatorname{det}\left(\mathcal{J}_{\mathbb{E}_{1}}-\lambda \mathrm{I}\right)=\left(d_{2 r}-d_{r}-h_{2 r}(u)-\lambda\right)\left(g_{2}-g-h_{2}(u)-\lambda\right)\left(d_{2}-d-\lambda\right) \operatorname{det} C_{1}
$$

where

$$
C_{1}=\left(\begin{array}{ccc}
d_{0}\left(\frac{n}{d_{0}}-1\right)-\lambda & d_{0}\left(\frac{n}{d_{0}}-1\right)-\lambda & d_{0}\left(\frac{n}{d_{0}}-1\right)-\lambda \\
0 & \frac{m c_{x} d_{0}}{c_{x} d_{0}+c_{y}\left(n-d_{0}\right)}-g_{0}-\lambda & 0 \\
0 & 0 & \frac{m c_{x} d_{0}}{c_{x} d_{0}+c_{y}\left(n-d_{0}\right)}-g_{0 r}-\lambda
\end{array}\right) .
$$

We have

$$
\begin{aligned}
\operatorname{det}\left(\mathcal{J}_{\mathbb{E}_{1}}-\lambda \mathbf{I}\right)= & \left(d_{2 r}-d_{r}-h_{2 r}(u)-\lambda\right)\left(g_{2}-g-h_{2}(u)-\lambda\right)\left(d_{2}-d-\lambda\right) \\
& \times\left(\frac{m c_{x} d_{0}}{c_{x} d_{0}+c_{y}\left(n-d_{0}\right)}-g_{0}-\lambda\right)\left(\frac{m c_{x} d_{0}}{c_{x} d_{0}+c_{y}\left(n-d_{0}\right)}-g_{0 r}-\lambda\right) \\
& \times\left(d_{0}\left(\frac{n}{d_{0}}-1\right)-\lambda\right) .
\end{aligned}
$$

Then, the eigenvalues are $\lambda_{1}=d_{2 r}-d_{r}-h_{2 r}(u), \quad \lambda_{2}=g_{2}-g-h_{2}(u), \quad \lambda_{3}=d_{2}-d$, $\lambda_{4}=\frac{m c_{x} d_{0}}{c_{x} d_{0}+c_{y}\left(n-d_{0}\right)}-g_{0}, \lambda_{5}=\frac{m c_{x} d_{0}}{c_{x} d_{0}+c_{y}\left(n-d_{0}\right)}-g_{0 r}$ and $\lambda_{6}=d_{0}\left(\frac{n}{d_{0}}-1\right)$. 
3. For the equilibrium point $\mathbb{E}_{2}$ we have

$$
\mathcal{J}_{\mathbb{E}_{2}}=\left(\begin{array}{cccccc}
a_{11} & 0 & a_{13} & 0 & a_{15} & 0 \\
r & d_{2}-d & 0 & 0 & 0 & 0 \\
a_{13} & 0 & a_{33} & 0 & a_{35} & 0 \\
0 & 0 & q & g_{2}-g-h_{2}(u) & 0 & 0 \\
0 & 0 & 0 & 0 & g_{0}-g_{0 r} & 0 \\
0 & 0 & 0 & 0 & q & d_{2 r}-d_{r}-h_{2 r}(u)
\end{array}\right)
$$

such that

$$
\begin{aligned}
& a_{11}=\frac{d_{0}^{2}}{(1-\alpha) n c_{y}}\left[\alpha c_{y}\left(\frac{n}{d_{0}}-1\right)-c_{x}\left(\frac{m}{g_{0}}-1\right)\right], \\
& a_{13}=\frac{d_{0}^{2}}{(1-\alpha) n c_{y}}\left[\alpha c_{y}\left(\frac{n}{d_{0}}-1\right)-c_{x}\left(\frac{m}{g_{0}}-1\right)\right], \\
& a_{15}=\frac{\alpha d_{0}^{2}}{(1-\alpha) n}\left(\frac{n}{d_{0}}-1\right)-\frac{d_{0}^{2} c_{x}}{(1-\alpha) n c_{y}}\left(\frac{m}{g_{0}}-1\right), \\
& a_{31}=\frac{g_{0}^{2}}{(1-\alpha) m c_{x}}\left[c_{x}\left(\frac{m}{g_{0}}-1\right)-c_{y}\left(\frac{n}{d_{0}}-1\right)\right], \\
& a_{33}=\frac{\alpha g_{0}^{2}}{(1-\alpha) m c_{x}}\left[c_{x}\left(\frac{m}{g_{0}}-1\right)-c_{y}\left(\frac{n}{d_{0}}-1\right)\right], \\
& a_{35}=\frac{\alpha g_{0}^{2}}{(1-\alpha) m c_{x}}\left[c_{x}\left(\frac{m}{g_{0}}-1\right)-c_{y}\left(\frac{n}{d_{0}}-1\right)\right], \\
& a_{55}=g_{0}-g_{0 r},
\end{aligned}
$$$$
\operatorname{det}\left(\mathcal{J}_{\mathbb{E}_{2}}-\lambda \mathrm{I}\right)=\left(d_{2 r}-d_{r}-h_{2 r}(u)-\lambda\right)\left(g_{2}-g-h_{2}(u)-\lambda\right)\left(d_{2}-d-\lambda\right) \operatorname{det} C_{2}
$$

and

$$
\operatorname{det} C_{2}=\left(a_{55}-\lambda\right)\left[\lambda^{2}-\left(a_{11}+a_{33}\right) \lambda+a_{11} a_{33}-a_{13} a_{31}\right] \text {. }
$$

We note that $a_{55}$ is a positive eigenvalue, then $\mathbb{E}_{2}$ is unstable.

4. For the equilibrium point $\mathbb{E}_{3}$ we have

$$
\mathcal{J}_{\mathbb{E}_{3}}=\left(\begin{array}{cccccc}
a_{11} & 0 & a_{13} & 0 & a_{15} & 0 \\
r & d_{2}-d & 0 & 0 & 0 & 0 \\
0 & 0 & a_{33} & 0 & 0 & 0 \\
0 & 0 & q & g_{2}-g-h_{2}(u) & 0 & 0 \\
a_{51} & 0 & a_{53} & 0 & a_{55} & 0 \\
0 & 0 & 0 & 0 & q & d_{2 r}-d_{r}-h_{2 r}(u)
\end{array}\right)
$$


such that

$$
\begin{aligned}
& a_{11}=\frac{d_{0}^{2}}{(1-\alpha) n c_{y}}\left[\alpha c_{y}\left(\frac{n}{d_{0}}-1\right)-c_{x}\left(\frac{m}{g_{0 r}}-1\right)\right], \\
& a_{13}=\frac{d_{0}^{2}}{(1-\alpha) n c_{y}}\left[\alpha c_{y}\left(\frac{n}{d_{0}}-1\right)-c_{x}\left(\frac{m}{g_{0 r}}-1\right)\right], \\
& a_{15}=\frac{d_{0}^{2}}{(1-\alpha) n c_{y}}\left[\alpha c_{y}\left(\frac{n}{d_{0}}-1\right)-c_{x}\left(\frac{m}{g_{0 r}}-1\right)\right], \\
& a_{33}=g_{0 r}-g_{0}, \\
& a_{51}=\frac{g_{0 r}^{2}}{(1-\alpha) m c_{x}}\left[c_{x}\left(\frac{m}{g_{0 r}}-1\right)-c_{y}\left(\frac{n}{d_{0}}-1\right)\right], \\
& a_{53}=\frac{\alpha g_{0 r}^{2}}{(1-\alpha) m c_{x}}\left[c_{x}\left(\frac{m}{g_{0 r}}-1\right)-c_{y}\left(\frac{n}{d_{0}}-1\right)\right], \\
& a_{55}=\frac{\alpha g_{0 r}^{2}}{(1-\alpha) m c_{x}}\left[c_{x}\left(\frac{m}{g_{0 r}}-1\right)-c_{y}\left(\frac{n}{d_{0}}-1\right)\right],
\end{aligned}
$$

$$
\operatorname{det}\left(\mathcal{J}_{\mathbb{E}_{3}}-\lambda \mathrm{I}\right)=\left(d_{2 r}-d_{r}-h_{2 r}(u)-\lambda\right)\left(g_{2}-g-h_{2}(u)-\lambda\right)\left(d_{2}-d-\lambda\right) \operatorname{det} C_{3}
$$

and

$$
\operatorname{det} C_{3}=\left(a_{33}-\lambda\right)\left[\lambda^{2}-\left(a_{11}+a_{55}\right) \lambda+a_{11} a_{55}-a_{15} a_{51}\right] .
$$

According to the conditions of existence of the equilibrium point $\mathbb{E}_{3}$ we can prove that one of eigenvalues is positive that is $\mathbb{E}_{3}$ is unstable.

5. For the equilibrium point $\mathbb{E}_{4}$ we have

$$
\begin{gathered}
\mathcal{J}_{\mathbb{E}_{4}}=\left(\begin{array}{cccccc}
\frac{n \alpha c_{y} g_{0}}{\alpha c_{y} g_{0}+c_{x}\left(m-g_{0}\right)}-d_{0} & 0 & 0 & 0 & 0 & 0 \\
r & d_{2}-d & 0 & 0 & 0 & 0 \\
\frac{g_{0}}{\alpha}\left(\frac{g_{0}}{m}-1\right) & 0 & g_{0}\left(\frac{g_{0}}{m}-1\right) & 0 & g_{0}\left(\frac{g_{0}}{m}-1\right) & 0 \\
0 & 0 & q & g_{2}-g-h_{2}(u) & 0 & 0 \\
0 & 0 & 0 & 0 & g_{0}-g_{0 r} & 0 \\
0 & 0 & 0 & 0 & q & d_{2 r}-d_{r}-h_{2 r}(u)
\end{array}\right), \\
\operatorname{det}\left(\mathcal{J}_{\mathbb{E}_{4}}-\lambda \mathrm{I}\right)=\left(d_{2 r}-d_{r}-h_{2 r}(u)-\lambda\right)\left(g_{2}-g-h_{2}(u)-\lambda\right)\left(d_{2}-d-\lambda\right) \operatorname{det} C_{4}
\end{gathered}
$$

and

$$
C_{4}=\left(\begin{array}{ccc}
\frac{n \alpha c_{y} g_{0}}{\alpha c_{y} g_{0}+c_{x}\left(m-g_{0}\right)}-d_{0}-\lambda & 0 & 0 \\
\frac{g_{0}}{\alpha}\left(\frac{g_{0}}{m}-1\right) & g_{0}\left(\frac{g_{0}}{m}-1\right)-\lambda & g_{0}\left(\frac{g_{0}}{m}-1\right) \\
0 & 0 & g_{0}-g_{0 r}-\lambda
\end{array}\right) .
$$


We have

$$
\begin{aligned}
\operatorname{det}\left(\mathcal{J}_{\mathbb{E}_{4}}-\lambda \mathrm{I}\right)= & \left(d_{2 r}-d_{r}-h_{2 r}(u)-\lambda\right)\left(g_{2}-g-h_{2}(u)-\lambda\right)\left(d_{2}-d-\lambda\right) \\
& \left(\frac{n \alpha c_{y} g_{0}}{\alpha c_{y} g_{0}+c_{x}\left(m-g_{0}\right)}-d_{0}-\lambda\right)\left(g_{0}\left(\frac{g_{0}}{m}-1\right)-\lambda\right)\left(g_{0}-g_{0 r}-\lambda\right) .
\end{aligned}
$$

Note that $\lambda=g_{0}-g_{0 r}$ is a positive eigenvalue which implies that $\mathbb{E}_{4}$ is unstable.

6. For the equilibrium point $\mathbb{E}_{5}$ we have

$$
\begin{aligned}
& \mathcal{J}_{\mathbb{E}_{5}}=\left(\begin{array}{cccccc}
\frac{n \alpha c_{y} g_{0 r}}{\alpha c_{y} g_{0 r}+c_{x}\left(m-g_{0 r}\right)}-d_{0} & 0 & 0 & 0 & 0 & 0 \\
r & d_{2}-d & 0 & 0 & 0 & 0 \\
0 & 0 & g_{0 r}-g_{0} & 0 & 0 & 0 \\
0 & 0 & q & g_{2}-g-h_{2}(u) & 0 & 0 \\
\frac{g_{0 r}}{\alpha}\left(\frac{g_{0 r}}{m}-1\right) & 0 & g_{0 r}\left(\frac{g_{0 r}}{m}-1\right) & 0 & g_{0 r}\left(\frac{g_{0 r}}{m}-1\right) & 0 \\
0 & 0 & 0 & 0 & q & d_{2 r}-d_{r}-h_{2 r}(u)
\end{array}\right), \\
& \operatorname{det}\left(\mathcal{J}_{\mathbb{E}_{5}}-\lambda \mathrm{I}\right)=\left(d_{2 r}-d_{r}-h_{2 r}(u)-\lambda\right)\left(g_{2}-g-h_{2}(u)-\lambda\right)\left(d_{2}-d-\lambda\right) \operatorname{det} C_{5}
\end{aligned}
$$

and

$$
C_{5}=\left(\begin{array}{ccc}
\frac{n \alpha c_{y} g_{0 r}}{\alpha c_{y} g_{0 r}+c_{x}\left(m-g_{0 r}\right)}-d_{0}-\lambda & 0 & 0 \\
0 & g_{0 r}-g_{0}-\lambda & 0 \\
\frac{g_{0 r}}{\alpha}\left(\frac{g_{0 r}}{m}-1\right) & g_{0 r}\left(\frac{g_{0 r}}{m}-1\right) g_{0 r}\left(\frac{g_{0 r}}{m}-1\right)-\lambda
\end{array}\right) .
$$

We have

$$
\begin{aligned}
\operatorname{det}\left(\mathcal{J}_{\mathbb{E}_{5}}-\lambda \mathrm{I}\right)= & \left(d_{2 r}-d_{r}-h_{2 r}(u)-\lambda\right)\left(g_{2}-g-h_{2}(u)-\lambda\right)\left(d_{2}-d-\lambda\right) \\
& \left(\frac{n \alpha c_{y} g_{0 r}}{\alpha c_{y} g_{0 r}+c_{x}\left(m-g_{0 r}\right)}-d_{0}-\lambda\right)\left(g_{0 r}-g_{0}-\lambda\right)\left(g_{0 r}\left(\frac{g_{0 r}}{m}-1\right)-\lambda\right) .
\end{aligned}
$$

Then the eigenvalues are $\lambda_{1}=d_{2 r}-d_{r}-h_{2 r}(u), \quad \lambda_{2}=g_{2}-g-h_{2}(u), \quad \lambda_{3}=d_{2}-d$, $\lambda_{4}=\frac{n \alpha c_{y} g_{0 r}}{\alpha c_{y} g_{0 r}+c_{x}\left(m-g_{0 r}\right)}-d_{0}, \lambda_{5}=g_{0 r}-g_{0}$ and $\lambda_{6}=g_{0 r}\left(\frac{g_{0 r}}{m}-1\right)$.

\section{Numerical Simulation for the local stability}

In this section, we give some numerical simulations for the points $E_{1}$ and $E_{5}$ to illustrate our results. 


\section{WMLS 2014}

\subsection{The equilibrium point $E_{1}$}

The equilibrium $E_{1}$ corresponds to the situation where the cancer disappears, and it is important to obtain conditions of stability of $E_{1}$ in order to stop the onset of the diseases, in Figs. (1-6) we give numerical simulations for $E_{1}$.

Figures 1-6 correspond to the case where conditions of stability of $E_{1}$ are satisfied.

\begin{tabular}{|c|c|}
\hline The parameters & values \\
\hline$n$ & 50 \\
\hline$m$ & 20 \\
\hline$\alpha$ & 0.1 \\
\hline$q$ & $1.065 \times 10^{7}$ \\
\hline$d$ & 9.4 \\
\hline$d_{0}$ & 20 \\
\hline$d_{2}$ & 0.4 \\
\hline$d_{r}$ & 1.4 \\
\hline$d_{2 r}$ & 0.5 \\
\hline$c_{x}$ & $0.75 \times 10^{-4}$ \\
\hline$c_{y}$ & $0.38 \times 10^{-4}$ \\
\hline$g$ & 1.4 \\
\hline$g_{0}$ & 25 \\
\hline$g_{0 r}$ & 20 \\
\hline$g_{2}$ & 0.8 \\
\hline$h_{2}$ & 0.7 \\
\hline$h_{2 r}$ & 0.3 \\
\hline$r$ & $1.065 \times 10^{7}$ \\
\hline
\end{tabular}

With the starting point $\left(20000.01,\left(2.3667 \times 10^{10}\right)+0.01,0.01,0.01,0.01,0.01\right)$.

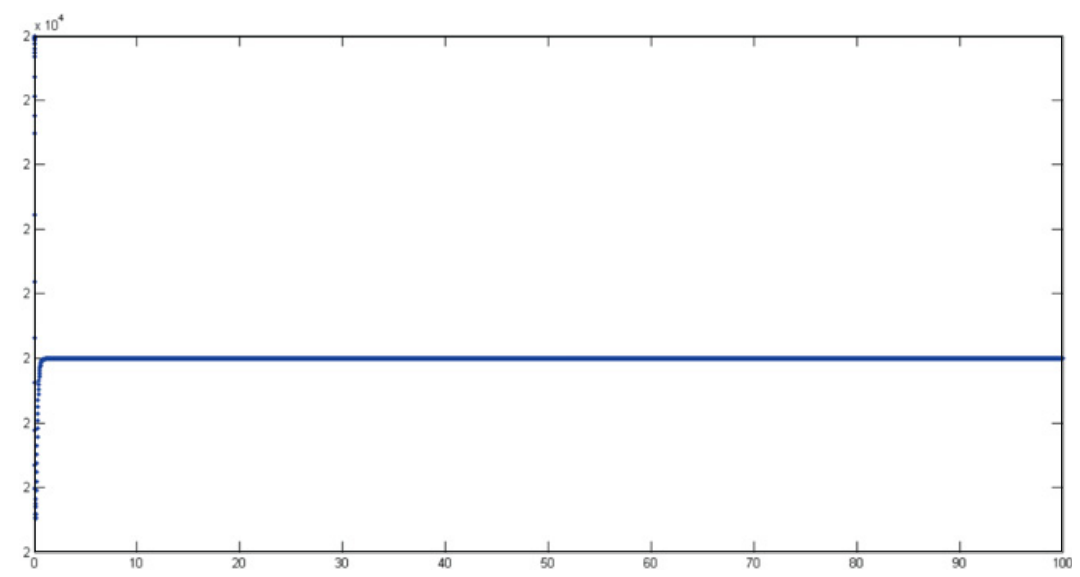

Figure 1. Behavior of normal stem cells $x_{0}$. 
ITM Web of Conferences

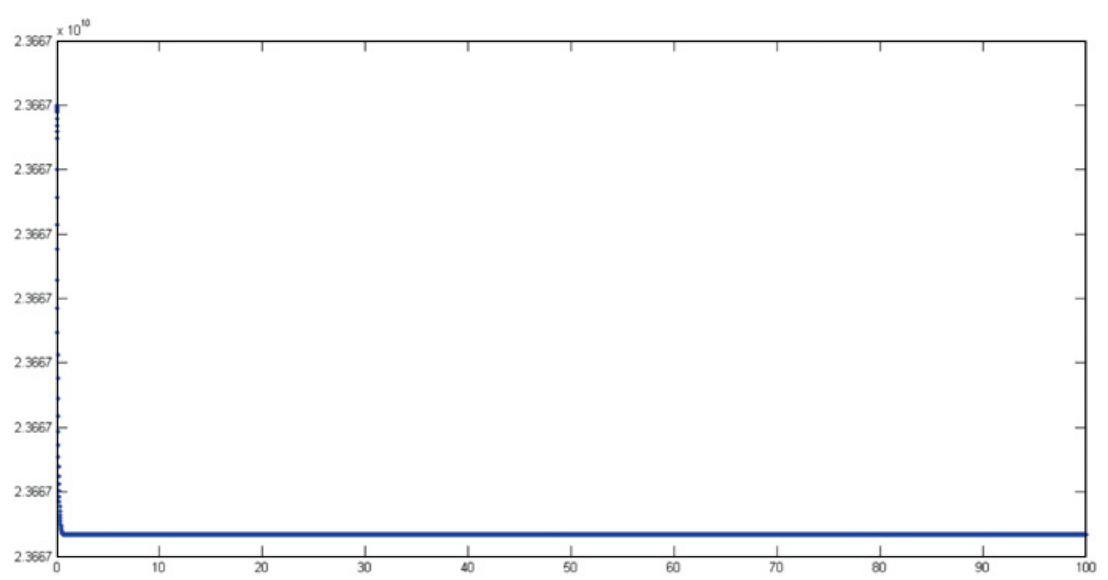

Figure 2. Behavior of normal differentiated cells $x_{1}$.

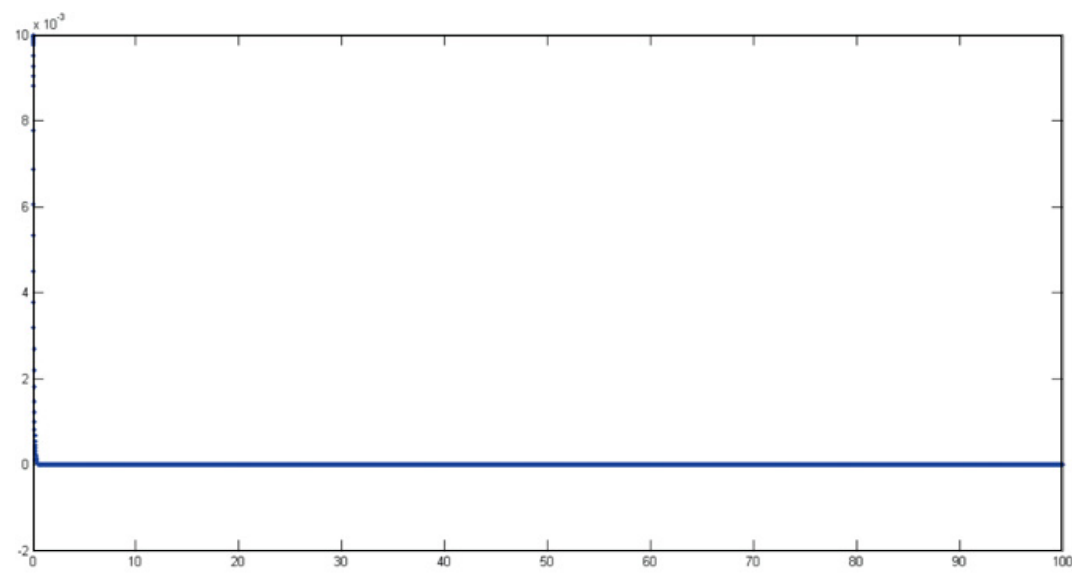

Figure 3. Behavior of sensitive cancerous stem cells $y_{0}$.

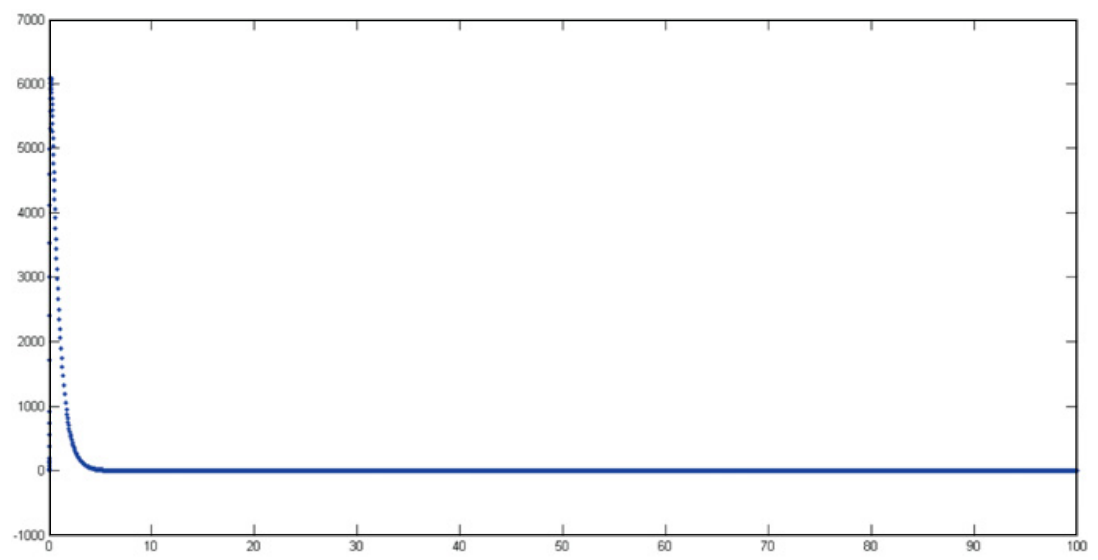

Figure 4. Behavior of sensitive cancerous differentiated cells $y_{1}$. 
WMLS 2014

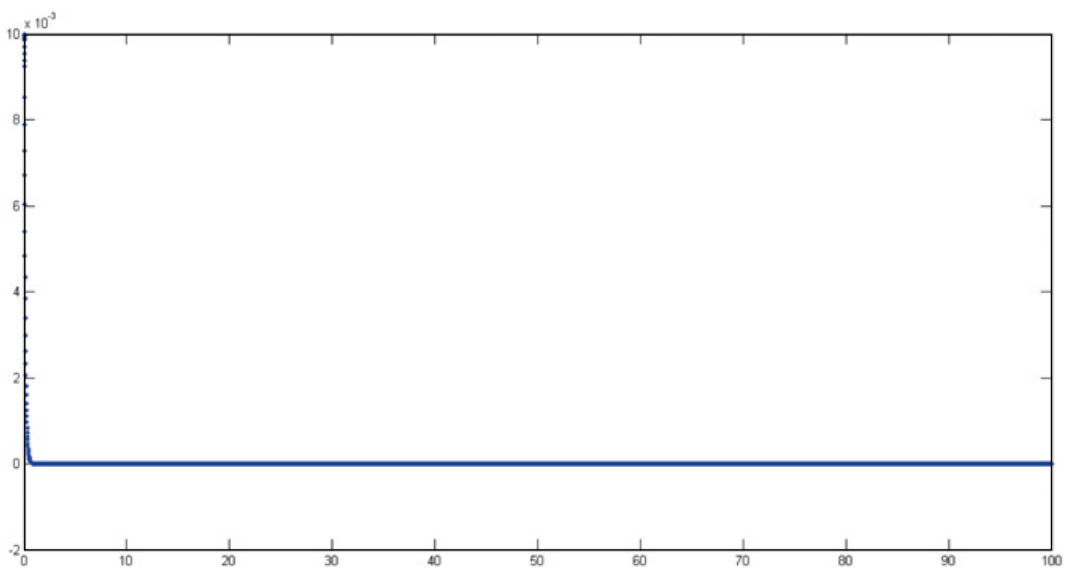

Figure 5. Behavior of resistant cancerous stem cells $z_{0}$.

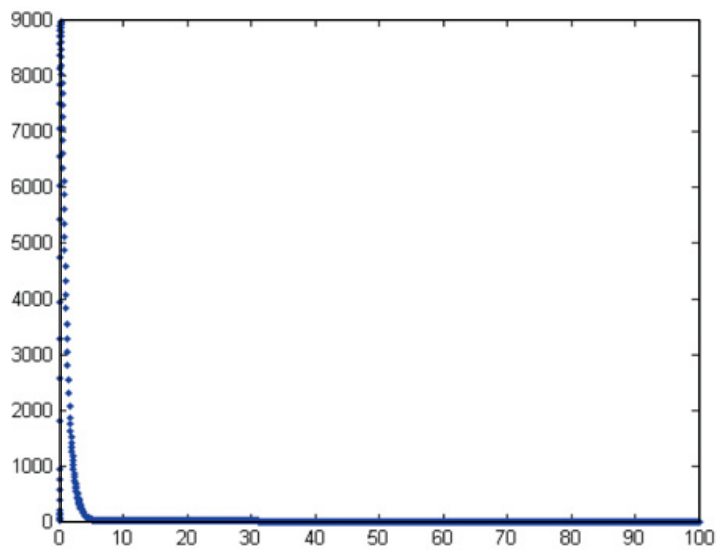

Figure 6. Behavior of resistant cancerous differentiated cells $z_{1}$. 


\subsection{The equilibrium point $\boldsymbol{E}_{5}$}

The equilibrium point $E_{5}$ corresponds to limit situation where normal and sensitive cancerous cells are eliminated and all remaining cells are resistant cancerous cells. (see Figs. 7-9).

\begin{tabular}{|c|c|}
\hline The parameters & values \\
\hline$n$ & 50 \\
\hline$m$ & 45 \\
\hline$\alpha$ & 0.1 \\
\hline$q$ & $1.065 \times 10^{7}$ \\
\hline$d$ & 9.4 \\
\hline$d_{0}$ & 60 \\
\hline$d_{2}$ & 0.4 \\
\hline$d_{r}$ & 1.4 \\
\hline$d_{2 r}$ & 0.5 \\
\hline$c_{x}$ & $0.75 \times 10^{-4}$ \\
\hline$c_{y}$ & $0.38 \times 10^{-4}$ \\
\hline$g$ & 1.4 \\
\hline$g_{0}$ & 46 \\
\hline$g_{0 r}$ & 40 \\
\hline$g_{2}$ & 0.8 \\
\hline$h_{2}$ & 0.7 \\
\hline$h_{2 r}$ & 0.3 \\
\hline$r$ & $1.065 \times 10^{7}$ \\
\hline
\end{tabular}

With the starting point $(0.0001,0.0001,0.0001,0.0001,0.0001475,421.5626)$.
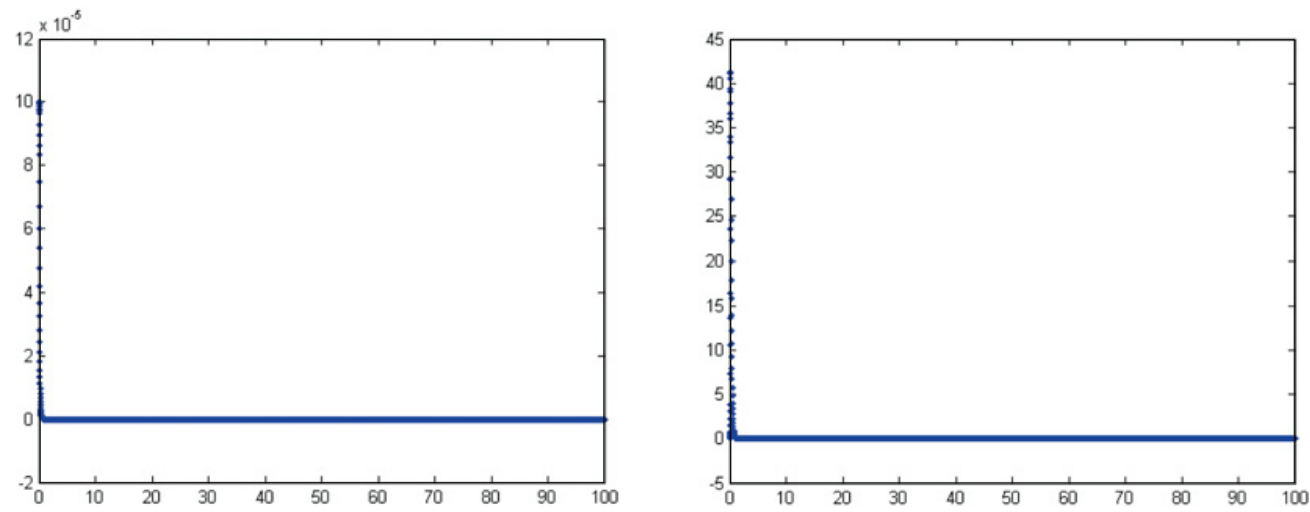

Figure 7. Behavior of normal stem and differentiated cells $x_{0}$ and $x_{1}$. 


\section{WMLS 2014}
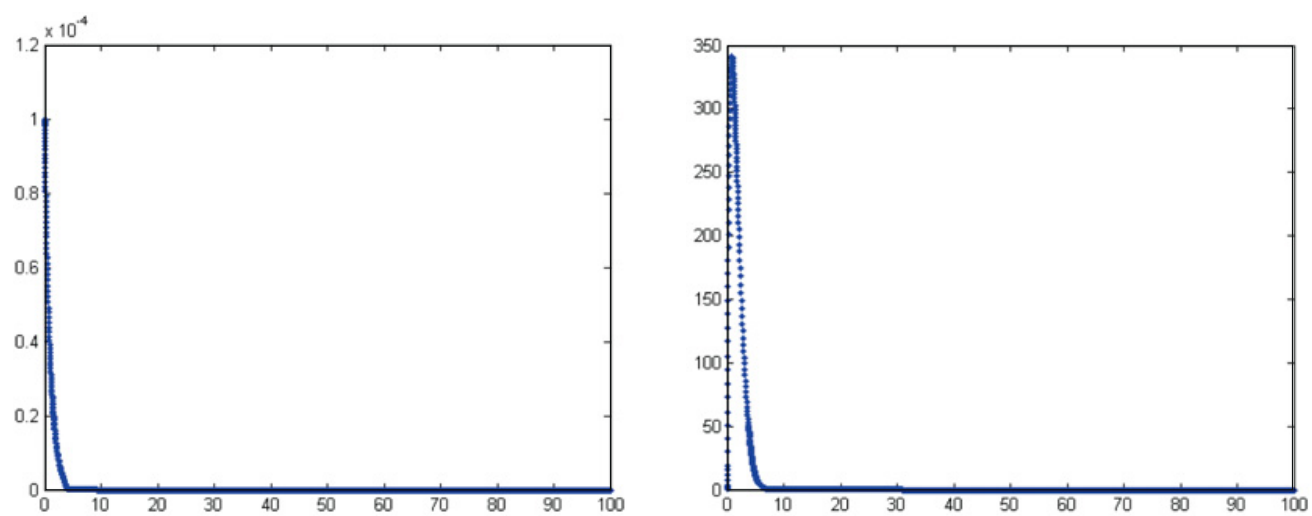

Figure 8. Behavior of sensitive cancerous stem and differentiated cells $y_{0}$ and $y_{1}$.
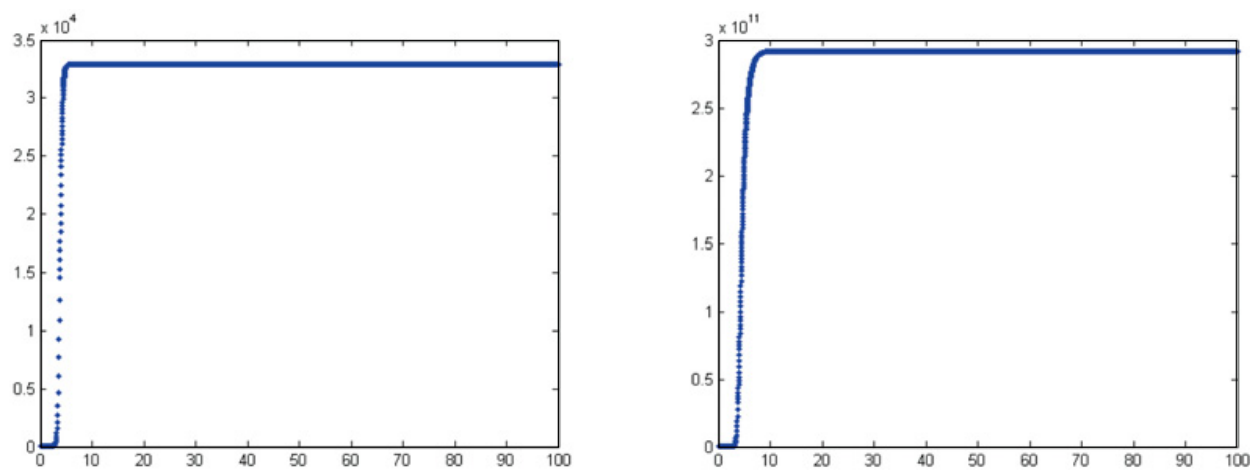

Figure 9. Behavior of resistant cancerous stem and differentiated cells $z_{0}$ and $z_{1}$.

\section{References}

[1] M. Adimy, F. Crauste and S. Ruan, A mathematical Study of the hematopoiesis process with applications to chronic myelogenous leukemia, SIAM Journal on Applied Mathematics, 65 (2005) 1328-1352.

[2] D. Dingli and F. Michor, Successful therapy must eradicate cancer stem cells, stem cells, 24 (2006) 2603-2610.

[3] M. C. Mackey, A unified hypothesis for the origin of aplastic anemia and periodic hematopoiesis. Blood, 51 (1978) 941-956.

[4] F. Michor, T. P. Hughes, Y. Iwasa, S. Branford, N. Shah, C.L. Sawyers and M.A. Nowak, Dynamics of chronic myeloid leukemia. Nature 435 (2005) 1267-1270.

[5] G. Volpe, C. Panuzzo, S. Ulisciani and D. Cilloni, Imatinib resistance in CML. Cancer Letters 274 (2009) 1-9. 